\title{
Distributed Recursive Filtering for Stochastic Systems under Uniform Quantizations and Deception Attacks through Sensor Networks *
}

\author{
Derui Ding ${ }^{\mathrm{a}, *}$, Zidong Wang ${ }^{\mathrm{b}}$, Daniel W. C. Ho ${ }^{\mathrm{c}}$, Guoliang Wei ${ }^{\mathrm{a}}$ \\ a Shanghai Key Lab of Modern Optical System, Department of Control Science and Engineering, University of Shanghai for \\ Science and Technology, Shanghai 200093, China. \\ ${ }^{\mathrm{b}}$ Department of Computer Science, Brunel University London, Uxbridge, Middlesex, UB8 3PH, U.K. \\ ${ }^{\mathrm{c}}$ Department of Mathematics, City University of Hong Kong, Kowloon, Hong Kong, China.
}

\begin{abstract}
This paper is concerned with the distributed recursive filtering problem for a class of discrete time-delayed stochastic systems subject to both uniform quantization and deception attack effects on the measurement outputs. The target plant is disturbed by the multiplicative as well as additive white noises. A novel distributed filter is designed where the available innovations are from not only the individual sensor but also its neighbouring ones according to the given topology. Attention is focused on the design of a distributed recursive filter such that, in the simultaneous presence of time-delays, deception attacks and uniform quantization effects, an upper bound for the filtering error covariance is guaranteed and subsequently minimized by properly designing the filter parameters via a gradient-based method at each sampling instant. Furthermore, by utilizing the mathematical induction, a sufficient condition is established to ensure the asymptotic boundedness of the sequence of the error covariance. Finally, a simulation example is utilized to illustrate the usefulness of the proposed design scheme of distributed filters.
\end{abstract}

Key words: Sensor networks; Distributed filtering; Recursive filtering; Deception attacks; Uniform quantization.

\section{Introduction}

For a few decades, as one of the most notable algorithms for state estimation problems, the Kalman filtering ( $\mathrm{K}$ F) technique has been playing an important role in signal processing and system control fields $[2,3,36]$. The $\mathrm{KF}$ technique is essentially a maximum-likelihood estimate algorithm for linear models with Gaussian noises under a quadratic performance criterion [12]. To cope with nonlinearities and/or uncertainties, there have been a few sub-optimal variants based on the traditional K$\mathrm{F}$ algorithm. Examples include, but are not limited to, the robust $\mathrm{KF}$ for uncertain systems [12], the extended $\mathrm{KF}$ (EKF) or the unscented KF for nonlinear system$\mathrm{s}$ [15] and the $\mathrm{KF} / \mathrm{EKF}$ for systems with equality constraints [29]. Recently, the Kalman filtering problem for

\footnotetext{
* This work was supported in part by the Royal Society of the UK, the National Natural Science Foundation of China under Grants 61329301, 61573246 and 61374039, the Research Grants Council of Hong Kong Special Administrative Region under grant GRF 11300415 and a grant from CityU 7004672, the Shanghai Rising-Star Program of China under Grant 16QA1403000, the Program for Capability Construction of Shanghai Provincial Universities under Grant 15550502500, and the Alexander von Humboldt Foundation of Germany.

* Tel.: +86 21 55271299; fax: +86 2155271299.

Email addresses: deruiding2010@usst.edu.cn (Derui Ding), Zidong.Wang@brunel.ac.uk (Zidong Wang), madaniel@cityu.edu.hk (Daniel W. C. Ho), guoliang. wei1973@gmail.com (Guoliang Wei).
}

systems with communication delays has received considerable research interest corresponding to the popularity of the networked systems. In case of the discrete timedelayed systems, the filtering algorithms have been proposed via system augmentation approach in order to utilize the Riccati equation methods [22]. Different from the discrete-time case, the continuous-time system with delayed measurements can be converted into a nominal system with delay-free measurements via the re-organizing method [18]. It is worth mentioning that the computational burden would become an issue for the augmentation and re-organizing methods especially when the time-delays are relatively large. Obviously, an adequate trade-off between the estimation accuracy and the computational efficiency should be taken into account when dealing with time-delays in the filtering problems.

It is well known that, in networked systems, an analogto-digital converter is usually adopted to convert the continuous-time analog signal to the corresponding discrete-time digital one. Due primarily to the operation of rounding or truncation, the unavoidable difference between the actual analog value and converted digital one is customarily referred to as the quantization error or quantization distortion. This kind of errors can be modeled as a bounded unknown disturbance or as an additional random signal obeying uniform distribution $[4,28]$. Up to now, the stabilization and minimum data transmission rate issues with uniform quantization have been investigated by utilizing Lyapunov functions combined with perturbation analysis techniques, see e.g. [4]. It is worth emphasizing that the quantization errors from uniform quantizers are non-square summable 
sequences that cannot be described by the widely used state-dependent Lipschitz-like conditions [20] and therefore cannot be handled by the well-known robust design techniques. This may well explain why the results on filter/controller design problems under uniform quantizations have been scattered in comparison with those for logarithmic quantizations.

On the other hand, in parallel with the quiet evolution of sensor network technologies, the distributed filtering problem through sensor networks has gained an everincreasing interest from researchers in many areas such as signal processing and control engineering, and a number of filtering algorithms have been proposed in the literature, see e.g. $[8,9,19]$ for the distributed $H_{\infty}$ filtering schemes and $[25]$ for the distributed KF methods. In particular, a distributed filtering framework that sheds insightful light on this domain has been established in [25] with the aid of two identical consensus filters for fusion of the sensor data and covariance information. A typical feature with the distributed filters is their collaborative information processing mechanism, that is, the information available on an individual node is not only from its own measurement but also from its neighboring sensors' measurements according to the given topology via open networks. Note that the sensor nodes are usually made of low cost devices with low computing capacity and limited battery power. As such, the sensor networks might be vulnerable to cyber-attacks especially during the signal transmission $[24,37]$ and, accordingly, the emerging cyber-security issues have been raised that have quickly attracted much attention, see e.g. [26,31].

In the general context of networked control systems, so far, much progress has been made on the security control/filtering problems by employing the techniques of dynamic programming or Lyapunov stability theory, see e.g. [1,21] for denial-of-service (DoS) attacks and $[6,7,11,16,26]$ for deception attacks. However, when it comes to the distributed filtering issues over sensor networks, only a limited number of results have been available in the literature (see e.g. [24,32,37]). For instance, under binary hypotheses with quantized sensor observations, the optimal attacking distributions have been estimated in [24] to minimize the detection error exponent and the fraction of Byzantine sensors (i.e. compromised sensors for adversaries). Recently, in [37], the identification and categorization issues of attacked sensors have been discussed by utilizing the joint estimation of the statistical description of the attacks and the estimated parameter. Note that most existing results have been concerned with static target plants despite the fact that dynamic target plants are more often encountered in engineering practice, which is due probably to the difficulties in analyzing the dynamics in collaborative nature and spatial structure when designing distributed filters. In the research area of cyber-security, the success ratio of the launched attacks has recently become an emerging topic of research from the defenders' perspectives. The launched attacks by the adversaries may not always be successful for mainly three reasons: 1) only a relatively small amount of attacks could pass through the detectors (with anti-attack countermeasures) for systems equipped with protection devices or software; 2) the attacks cannot be persistently (or arbitrarily) launched by the adversaries due to unavoidable limited resource (e.g. energy); and 3) the attacks sent through the networks with limited bandwidth are subject to randomly fluctuated condition changes (e.g. network load, network congestion and network transmission rate) and therefore cannot arrive at the desired end. As such, from the viewpoint of the defending party, the successfully occurred cyber-attacks can be understood to be intermittent or random in implementation, and the corresponding issue of intermittently or randomly occurred cyber-attacks have been dealt with in $[1,37,38]$. For example, the maximal number of succession attacks has been investigated in [38] where a variation of the receding-horizon control law has been proposed to deal with the replay attacks and analyze the resulting system performance degradation. Nevertheless, the intermittent or random nature of the successfully occurred cyber-attacks has not received adequate attention yet for the distributed filtering problem of dynamic target plants, not to mention the case where the multiplicative/additive noises [13] and the uniform quantizations are also the concerns.

Summarizing the above discussions, the focus of this paper is on the parameter design and performance analysis of distributed recursive filtering with uniform quantization and intermittent deception attacks. We endeavor to answer the following questions: 1) how to design a distributed filter effectively fusing the unreliable data corrupted by noises, quantization errors and possible deception attacks? 2) how to develop an efficient filtering algorithm that would help reduce the computation burden resulting from time delays and the large number of sensor nodes? and 3) how to cope with the complicated coupling issues between the filtering errors and observed states in the performance analysis? The main contribution of this paper is threefold: 1) a novel structure of distributed filters is designed to adequately utilize the available innovations from not only itself (credible measurements) but also its neighbouring sensors which could be subject to deception attacks; 2) the developed filter design algorithm is of a form suitable for distributed recursive computation in online applications via solving two Riccati-like difference equations; and 3) a sufficient condition is proposed to show the asymptotic boundedness of the filtering error covariance through intensive stochastic analysis.

Notation The notation used here is fairly standard except where otherwise stated. $\mathbb{R}^{n}$ and $\mathbb{R}^{n \times m}$ denote, respectively, the $n$ dimensional Euclidean space and the set of all $n \times m$ real matrices. $I$ denotes the identity matrix of compatible dimension. The notation $X \geq Y$ (respectively, $X>Y$ ) where $X$ and $Y$ are symmetric matrices, means that $X-Y$ is positive semi-definite (respectively, positive definite). $M^{T}$ represents the transpose of $M . \mathbb{E}\{x\}$ stands for the expectation of stochastic variable $x$. $\|x\|$ describes the Euclidean norm of a vector $x$. The shorthand $\operatorname{diag}\left\{M_{1}, M_{2}, \cdots, M_{n}\right\}$ denotes a block diagonal matrix with diagonal blocks being the matrices $M_{1}, M_{2}, \cdots, M_{n}$.

\section{Problem Formulation and Preliminaries}

In this paper, the underlying sensor network has $n$ sensor nodes which are distributed in space according to a fixed network topology represented by a directed graph $\mathscr{G}=(\mathscr{V}, \mathscr{E}, \mathscr{H})$ of order $n$ with the set of nodes 
$\mathscr{V}=\{1,2, \cdots, n\}$, the set of edges $\mathscr{E} \in \mathscr{V} \times \mathscr{V}$, and the weighted adjacency matrix $\mathscr{H}=\left[h_{i j}\right]$ with nonnegative adjacency element $h_{i j}$. An edge of $\mathscr{G}$ is denoted by the ordered pair $(i, j)$. The adjacency elements associated with the edges of the graph are positive, i.e., $h_{j i}>0 \Longleftrightarrow(j, i) \in \mathscr{E}$, which means that sensor $i$ can obtain information from sensor $j$. The set of neighbors of node $i \in \mathscr{V}$ is denoted by $\mathcal{N}_{i}=\{j \in \mathscr{V}:(j, i) \in \mathscr{E}\}$. The in-degree of Node $i$ is defined as $\hbar_{\text {in }}^{i}=\sum_{j \in \mathcal{N}_{i}} h_{j i}$. In this paper, assume $\hbar_{\text {in }}^{i} \neq 0$ for all nodes.

Let the target plant be described by the following discrete-time stochastic system with multiplicative noises:

$$
\begin{aligned}
x_{k+1}= & \left(A_{0, k}+\sum_{s=1}^{r} \omega_{s, k} A_{s, k}\right) x_{k} \\
& +\left(A_{0, k}^{d}+\sum_{s=1}^{r} \omega_{s, k} A_{s, k}^{d}\right) x_{k-\tau}+B_{k} w_{k}
\end{aligned}
$$

with $n$ sensors modeled by

$$
\tilde{y}_{i, k}=\left(C_{0, k}+\varpi_{i, k} C_{i, k}\right) x_{k}+D_{k} v_{i, k}, i=1,2, \cdots, n
$$

where $x_{k} \in \mathbb{R}^{n_{x}}$ is the state of the target plant that cannot be observed directly, $\tilde{y}_{i, k} \in \mathbb{R}^{n_{y}}$ is the ideal measurement output (without quantization) from sensor $i . w_{k} \in \mathbb{R}^{s}$ and $v_{i, k} \in \mathbb{R}^{p}(i=1,2, \cdots, n)$ are the white noises with zero-mean and unity covariance, and are mutually uncorrelated in $k$ and $i . \omega_{s, k} \in \mathbb{R}$ $(s=1,2, \cdots, r)$ and $\varpi_{i, k} \in \mathbb{R}(i=1,2, \cdots, n)$ are multiplicative noises with zero-mean and unity variances, and are mutually uncorrelated in $k . r$ and $\tau$ are two known positive integers. $A_{s, k}, A_{s, k}^{d}(s=0,1, \cdots, r)$ and $C_{i, k}(i=0,1, \cdots, n)$ are known constant matrices with compatible dimensions.

In this paper, a uniform quantizer is taken into account. Assume that the overall quantizer range is $[-M, M]$ with $M>0$. The length of the quantizer level $u$ is defined as $u=2 M /\left(2^{b}-1\right)$ where $b$ is the number of bits for digital sensors. The uniform quantizer [28] is denoted by

$$
\bar{y}_{i, k}=Q\left(\tilde{y}_{i, k}\right)=\tilde{y}_{i, k}+q_{i, k}
$$

where $\bar{y}_{i, k}$ is the quantized signal and $q_{i, k} \in \mathbb{R}^{n_{y}}$ is the quantization error process. Here, $q_{i, k}$ is an additive white uniform distributed noise with each element being uniformly distributed in $[-0.5 u, 0.5 u]$. Obviously, the variance of such a quantization error process is $\frac{u^{2}}{12} I$.

As discussed in the introduction, from the defenders' perspectives, the successfully occurred cyber-attacks could be intermittent or random in the implementation. In this case, the signals during the network transmission are subject to deception attacks modeled as follows:

$$
y_{i, k}=Q\left(\tilde{y}_{i, k}\right)+\alpha_{i, k} \zeta_{i, k}
$$

where $y_{i, k}$ is the received signal by neighboring nodes, $\zeta_{i, k} \in \mathbb{R}^{n_{y}}$ stands for the signal sent by attackers that is described as $\zeta_{i, k}=-Q\left(\tilde{y}_{i, k}\right)+\xi_{k}$. Here, the non-zero $\xi_{k}$ satisfying $\left\|\xi_{k}\right\| \leq \delta$ is an arbitrary limited magnitude signal and the bound $\delta$ is a known positive scalar that can be estimated through statistical tests or specified by security requirements. The variable $\alpha_{i, k}$ is a Bernoulli distributed white sequence taking values on 0 or 1 with the following probabilities

$$
\operatorname{Prob}\left\{\alpha_{i, k}=0\right\}=1-\bar{\alpha}, \quad \operatorname{Prob}\left\{\alpha_{i, k}=1\right\}=\bar{\alpha}
$$

where $\bar{\alpha} \in[0,1]$ is a known constant.

In this paper, the Kalman-type recursive filter on Node $i$ is of the following form:

$$
\left\{\begin{array}{l}
\hat{x}_{i, k+1 \mid k}=A_{0, k} \hat{x}_{i, k \mid k}+A_{0, k}^{d} \hat{x}_{i, k-\tau \mid k-\tau} \\
\hat{x}_{i, k+1 \mid k+1}=\hat{x}_{i, k+1 \mid k}+\hbar_{i n}^{i} K_{i, k+1}^{1} \\
\quad \times\left(\bar{y}_{i, k+1}-C_{0, k+1} \hat{x}_{i, k+1 \mid k}\right)+K_{i, k+1}^{2} \\
\quad \times \sum_{j \in \mathcal{N}_{i}} h_{j i}\left(y_{j, k+1}-(1-\bar{\alpha}) C_{0, k+1} \hat{x}_{i, k+1 \mid k}\right)(5 \mathrm{~b})
\end{array}\right.
$$

where $\hat{x}_{i, k \mid k}$ and $\hat{x}_{i, k+1 \mid k}$ are, respectively, the state estimate and the one-step prediction at time $k$, and $K_{i, k+1}^{1}$ and $K_{i, k+1}^{2}$ are the filter parameters to be determined.

Remark 1 For distributed filtering problems, the information available on each node is not only from itself but also from its neighbors according to the given topology. From the problem addressed in this paper, the data received from any neighboring sensors might be unreliable due to the possible deception attacks. As such, the innovation in (5b) is divided into two parts, that is, $\hbar_{i n}^{i}\left(\bar{y}_{i, k+1}-\right.$ $\left.C_{0, k+1} \hat{x}_{i, k+1 \mid k}\right)$ regarding the data from the node itself and $\sum_{j \in \mathcal{N}_{i}} h_{j i}\left(y_{j, k+1}-(1-\bar{\alpha}) C_{0, k+1} \hat{x}_{i, k+1 \mid k}\right)$ accounting for the data from the neighboring nodes. Therefore, the proposed filter model (5b) can be utilized to effectively fuse the data from two different sources, thereby improving the filtering performance.

Let us denote the one-step prediction error and the filtering error on Node $i$ as $e_{i, k+1 \mid k}:=x_{k+1}-\hat{x}_{i, k+1 \mid k}$ and $e_{i, k \mid k}:=x_{k}-\hat{x}_{i, k \mid k}$, respectively. The objective of this paper is twofold:

R1) Design a Kalman-type filter of the form (5a) and (5b) such that, in the presence of deception attacks, an upper bound for the filtering error covariance is guaranteed, i.e., there exists a sequence of positive-definite matrices $\Pi_{i, k \mid k}$ satisfying

$$
\mathbb{E}\left\{e_{i, k \mid k} e_{i, k \mid k}^{T}\right\} \leq \Pi_{i, k \mid k}, \quad \forall k>0
$$

Furthermore, the sequence of upper bounds $\Pi_{i, k \mid k}$ is minimized by the designed filter parameters $K_{i, k+1}^{1}$ and $K_{i, k+1}^{2}$ through a recursive scheme;

R2) For designed filter parameters $K_{i, k+1}^{1}$ and $K_{i, k+1}^{2}$, find a condition under which the sequence $\Pi_{i, k \mid k}$ is asymptotically bounded as time tends to infinity.

\section{Distributed filter design}

In this section, by resorting to the stochastic analysis combined with some special matrix inequalities, a sufficient condition on the filter design is proposed by solving two Riccati-like difference equations in order to guarantee an upper bound of the filtering error covariance. 
Moreover, such an upper bound is minimized based on the designed filter. Before proceeding further, we introduce the following lemmas which will be needed for the derivation of our main results. In addition, all proofs of Lemmas and Theorems are moved to the appendixes for clarity of presentation.

Lemma 1 [30] Suppose that $X=X^{T}>0, \Phi_{k}(X)=$ $\Phi_{k}^{T}(X) \in \mathbb{R}^{n_{x} \times n_{x}}$ and $\Psi_{k}(X)=\Psi_{k}^{T}(X) \in \mathbb{R}^{n_{x} \times n_{x}}$. If there exists $Y=Y^{T}>X$ such that

$$
\Phi_{k}(Y) \geq \Phi_{k}(X), \quad \Psi_{k}(Y) \geq \Phi_{k}(Y)
$$

then the solutions $M_{k}$ and $N_{k}$ to the following difference equations

$$
M_{k+1}=\Phi_{k}\left(M_{k}\right), N_{k+1}=\Psi_{k}\left(N_{k}\right), M_{0}=N_{0}>0
$$

satisfy $M_{k} \leq N_{k}$.

Lemma 2 For the addressed system (1), the state covariance matrix $X_{k+1}=\mathbb{E}\left\{x_{k+1} x_{k+1}^{T}\right\}$ obeys the following inequality:

$$
\begin{aligned}
X_{k+1} \leq & \left(1+\varepsilon_{k}\right) \sum_{s=0}^{r} A_{s, k} X_{k} A_{s, k}^{T}+B_{k} B_{k}^{T} \\
& +\left(1+\varepsilon_{k}^{-1}\right) \sum_{s=0}^{r} A_{s, k}^{d} X_{k-\tau}\left(A_{s, k}^{d}\right)^{T} .
\end{aligned}
$$

In light of Lemma 2, the upper bounds of both the covariance matrix $P_{i, k+1 \mid k}$ of one-step prediction error and the filtering error covariance $P_{i, k+1 \mid k+1}$ are presented in the following theorem.

Theorem 1 For the addressed system (1) with measurements (3) suffering from attacks (4), the covariance matrix $P_{i, k+1 \mid k}$ of one-step prediction errors and the filtering error covariance $P_{i, k+1 \mid k+1}$ satisfy

$$
P_{i, k+1 \mid k} \leq \Pi_{i, k+1 \mid k}, \quad P_{i, k+1 \mid k+1} \leq \Pi_{i, k+1 \mid k+1}
$$

where

$$
\begin{aligned}
\Pi_{i, k+1 \mid k}= & \left(1+\varepsilon_{k}\right) A_{0, k} \Pi_{i, k \mid k} A_{0, k}^{T}+B_{k} B_{k}^{T} \\
& +\left(1+\varepsilon_{k}^{-1}\right) A_{0, k}^{d} \Pi_{i, k-\tau \mid k-\tau}\left(A_{0, k}^{d}\right)^{T} \\
& +\left(1+\varepsilon_{k}\right) \sum_{i=1}^{r} A_{i, k} X_{k} A_{i, k}^{T} \\
& +\left(1+\varepsilon_{k}^{-1}\right) \sum_{i=1}^{r} A_{i, k}^{d} X_{k-\tau}\left(A_{i, k}^{d}\right)^{T} \\
\Pi_{i, k+1 \mid k+1}= & \Psi_{i, k+1}^{0} \Pi_{i, k+1 \mid k} \Psi_{i, k+1}^{0 T}+K_{i, k+1}^{1} \Psi_{i, k+1}^{1} \\
& \times K_{i, k+1}^{1 T}+K_{i, k+1}^{2} \Psi_{i, k+1}^{23} K_{i, k+1}^{2 T}
\end{aligned}
$$

with

$$
\begin{aligned}
\tilde{s}_{i} & =\sum_{l=1}^{n} \sum_{j=1}^{n} \bar{\alpha}^{2} h_{l i} h_{j i}+\sum_{l=1}^{n}\left(\bar{\alpha}-\bar{\alpha}^{2}\right)\left(h_{l i}\right)^{2} \\
\Psi_{i, k+1}^{0} & =I-\hbar_{i n}^{i} K_{i, k+1}^{1} C_{0, k+1}-\hbar_{i n}^{i}(1-\bar{\alpha}) K_{i, k+1}^{2} C_{0, k+1} \\
\Psi_{i, k+1}^{1} & =\left(\hbar_{i n}^{i}\right)^{2}\left(C_{i, k+1} X_{k+1} C_{i, k+1}^{T}+D_{k+1} D_{k+1}^{T}+\frac{u^{2} I}{12}\right)
\end{aligned}
$$

$$
\begin{aligned}
\Psi_{i, k+1}^{2}= & \sum_{j=1}^{n}(1-\bar{\alpha})\left(h_{j i}\right)^{2}\left(\bar{\alpha}\left(1+\varepsilon_{k}\right) C_{0, k+1} X_{k+1} C_{0, k+1}^{T}\right. \\
& \left.+C_{j, k+1} X_{k+1} C_{j, k+1}^{T}\right) \\
\Psi_{i, k+1}^{3}= & \left(1+2 \varepsilon_{k}^{-1}\right) \tilde{s}_{i} \delta^{2} I \\
& +\sum_{j=1}^{n}(1-\bar{\alpha})\left(h_{j i}\right)^{2}\left(D_{k+1} D_{k+1}^{T}+\frac{u^{2} I}{12}\right) \\
\Psi_{i, k+1}^{23}= & \Psi_{i, k+1}^{2}+\Psi_{i, k+1}^{3}, \Pi_{i, j \mid j}=P_{i, j \mid j}(-\tau \leq j \leq 0) .
\end{aligned}
$$

Up to now, the upper bound for the filtering error covariance has been derived. We are now in a position to consider the filter gain design problem for the addressed problem. The following result can be accessible by using the gradient-based approach.

Theorem 2 For the addressed system (1) with measurements (3) suffering from attacks (4), the gain matrices of the recursive filter (5a) and (5b) are given as follows

$$
\begin{aligned}
K_{i, k+1}^{1}= & \hbar_{i n}^{i} \Pi_{i, k+1 \mid k} C_{0, k+1}^{T}\left(I-\left(\hbar_{i n}^{i}\right)^{2}(1-\bar{\alpha})^{2}\right. \\
& \left.\times\left(\mathcal{S}_{i, k+1}^{2}\right)^{-1} \mathcal{S}_{i, k+1}^{0}\right)\left(\Omega_{i, k+1}^{1}\right)^{-1} \\
K_{i, k+1}^{2}= & (1-\bar{\alpha}) \hbar_{i n}^{i} \Pi_{i, k+1 \mid k} C_{0, k+1}^{T}\left(I-\left(\hbar_{i n}^{i}\right)^{2}\right. \\
& \left.\times\left(\mathcal{S}_{i, k+1}^{1}\right)^{-1} \mathcal{S}_{i, k+1}^{0}\right)\left(\Omega_{i, k+1}^{2}\right)^{-1}
\end{aligned}
$$

where

$$
\begin{aligned}
\mathcal{S}_{i, k+1}^{0} & =C_{0, k+1} \Pi_{i, k+1 \mid k} C_{0, k+1}^{T} \\
\mathcal{S}_{i, k+1}^{1} & =\left(\hbar_{i n}^{i}\right)^{2} \mathcal{S}_{i, k+1}^{0}+\Psi_{i, k+1}^{1} \\
\mathcal{S}_{i, k+1}^{2} & =\left(\hbar_{i n}^{i}\right)^{2}(1-\bar{\alpha})^{2} \mathcal{S}_{i, k+1}^{0}+\Psi_{i, k+1}^{23} \\
\Omega_{i, k+1}^{1} & =\mathcal{S}_{i, k+1}^{1}-(1-\bar{\alpha})^{2}\left(\hbar_{i n}^{i}\right)^{4} \mathcal{S}_{i, k+1}^{0}\left(\mathcal{S}_{i, k+1}^{2}\right)^{-1} \mathcal{S}_{i, k+1}^{0} \\
\Omega_{i, k+1}^{2} & =\mathcal{S}_{i, k+1}^{2}-(1-\bar{\alpha})^{2}\left(\hbar_{i n}^{i}\right)^{4} \mathcal{S}_{i, k+1}^{0}\left(\mathcal{S}_{i, k+1}^{1}\right)^{-1} \mathcal{S}_{i, k+1}^{0} .
\end{aligned}
$$

Furthermore, the upper bound of the filtering error covariance $\Pi_{i, k+1 \mid k+1}$ is recursively calculated by Riccatilike difference equation (11).

Remark 2 In the above theorem, the desired filter gain matrices are obtained with the aid of the solution of Riccati-like difference equations. It is not difficult to see that, for $\bar{\alpha}=1$, the gain $K_{i, k+1}^{1}$ and $K_{i, k+1}^{2}$ will reduce to the case of a single node without neighbors $\left(K_{i, k+1}^{2}=0\right.$ in this case), which implies that the filter on each node will refuse to fuse the information from the neighboring nodes when the network is completely unreliable. Furthermore, it should be pointed out that these two gains are designed by minimizing the trace of an upper bound for the filtering error covariance due to the effect from time-delays, deception attacks and uniform quantization. As such, the proposed filter is only a suboptimal one.

\section{Boundedness analysis}

So far, we have derived an upper bound for the filtering error covariance and such an upper bound is subsequently minimized by properly designing the filter parameters via a gradient-based method at each sampling instant. In reality, we would also be interested in understanding 
when the sequence of the upper bounds is asymptotically bounded in order to evaluate the performance of the designed recursive filter. In this section, for obtained filter gains, we will propose a sufficient condition ensuring the boundedness of the sequence $\Pi_{i, k \mid k}$ with respect to the filtering error covariance. For this purpose, we firstly introduce the following crucial assumption and lemmas. Similar to Section 3, all proofs of Lemmas and Theorems are moved to the appendixes for clarity of presentation.

Assumption 1 There are positive real constants $\bar{f}_{i}, \bar{f}_{i}^{d}$, $\underline{b}, \bar{b}, \underline{d}, \bar{d}$ and $\bar{c}_{i}(i=0,1,2, \cdots, r)$ such that the system parameter matrices are bounded:

$$
\begin{aligned}
A_{i, k} A_{i, k}^{T} & \leq \bar{f}_{i} I, \quad A_{i, k}^{d} A_{i, k}^{d T} \leq \bar{f}_{i}^{d} I, \quad C_{i, k} C_{i, k}^{T} \leq \bar{c}_{i} I \\
\underline{b} I & \leq B_{k} B_{k}^{T} \leq \bar{b} I, \quad \underline{d} I \leq D_{k} D_{k}^{T} \leq \bar{d} I .
\end{aligned}
$$

Lemma 3 [23] Let $A$ and $B$ be two $n \times n$ symmetric matrices with their eigenvalues listed as follows:

$\lambda_{\max }(A)=\lambda_{1}(A) \geq \lambda_{2}(A) \geq \cdots \geq \lambda_{n}(A)=\lambda_{\min }(A)$,

$\lambda_{\max }(B)=\lambda_{1}(B) \geq \lambda_{2}(B) \geq \cdots \geq \lambda_{n}(B)=\lambda_{\min }(B)$.

Then, one has

$$
\begin{aligned}
\min _{1 \leq i \leq s}\left(\lambda_{k}(A)\right. & \left.+\lambda_{s+1-i}(B)\right) \geq \lambda_{s}(A+B) \\
& \geq \max _{s \leq i \leq n}\left(\lambda_{i}(A)+\lambda_{n+s-i}(B)\right), \quad 1 \leq s \leq n .
\end{aligned}
$$

Furthermore, when either $A$ or $B$ is positive definite, the following are true for any $s \leq n$ :

$$
\begin{gathered}
\min _{1 \leq i \leq s}\left(\lambda_{i}(A) \lambda_{s+1-i}(B)\right) \geq \lambda_{s}(A B) \\
\geq \max _{s \leq i \leq n}\left(\lambda_{i}(A) \lambda_{n+s-i}(B)\right), \\
\min _{1 \leq i \leq s}\left(\lambda_{i}(A) \lambda_{s+1-i}\left(B B^{T}\right)\right) \geq \lambda_{s}\left(B A B^{T}\right) \\
\geq \max _{s \leq i \leq n}\left(\lambda_{i}(A) \lambda_{n+s-i}\left(B B^{T}\right)\right) .
\end{gathered}
$$

The following lemma is easily accessible by using the mathematical induction method and its proof is therefore omitted.

Lemma 4 Let $X_{i} \leq \bar{p}_{x} I(-\tau \leq i \leq 0)$ hold for the given positive scalar $\bar{p}_{x}$. For the addressed system (1), if there exists a positive scalar $\varepsilon$ satisfying

$$
\left((1+\varepsilon) \sum_{i=0}^{r} \bar{f}_{i}+\left(1+\varepsilon^{-1}\right) \sum_{i=0}^{r} \bar{f}_{i}^{d}\right) \bar{p}_{x}+\bar{b} \leq \bar{p}_{x},
$$

then $\underline{p}_{x} I \leq X_{k} \leq \bar{p}_{x} I$ is true for all $k>0$ where $\underline{p}_{x}$ is a known positive scalar satisfying $\underline{p}_{x} \leq \underline{b}$.

Now, let us give the main result, whose proof is moved to the appendix for clarity of presentation.

Theorem 3 Under Assumption 1, let $\Pi_{i, j \mid j} \leq \bar{p}_{\pi_{i}} I$ $(-\tau \leq j \leq 0)$ where $\bar{p}_{\pi_{i}}$ is a known positive scalar. For the addressed system (1) with the filtering dynamics (5a)-(5b), $\Pi_{i, k+1 \mid k+1} \leq \bar{p}_{\pi_{i}}$ I holds for any $k \geq 1$ if there exists a positive scalar $\varepsilon$ satisfying

$$
\left(3+\xi_{0}\right) \varrho_{1} \leq \bar{p}_{\pi_{i}}
$$

where

$$
\begin{aligned}
\xi_{0}= & \frac{\left(\hbar_{i n}^{i} \phi_{i, 1}^{m \Psi}\right)^{2} \bar{c}_{0} \varrho_{1}}{\left(\phi_{i, 1}^{n \Psi}\right)^{3}}+\frac{\left(\hbar_{i n}^{i} \phi_{i, 23}^{m \Psi}\right)^{2}(1-\bar{\alpha})^{2} \bar{c}_{0} \varrho_{1}}{\left(\phi_{i, 23}^{n \Psi}\right)^{3}} \\
& +\frac{3\left(\hbar_{i n}^{i}\right)^{4} \bar{c}_{0}^{2} \varrho_{1}^{2}}{\left(\phi_{i, 1}^{n \Psi}\right)^{2}}+\frac{3\left(\hbar_{i n}^{i}\right)^{4} \bar{c}_{0}^{2} \varrho_{1}^{2}}{\left(\phi_{i, 23}^{n \Psi}\right)^{2}} \\
\phi_{i, 1}^{m \Psi}= & \left(\hbar_{i n}^{i}\right)^{2}\left(\bar{C}_{i} \bar{p}_{x}+\bar{d}+u^{2} / 12\right), \phi_{i, 1}^{n \Psi}=\left(\hbar_{i n}^{i} u\right)^{2} / 12 \\
\phi_{i, 23}^{m \Psi}= & \sum_{j=1}^{n}(1-\bar{\alpha})\left(h_{j i}\right)^{2}\left(\bar{\alpha}(1+\varepsilon) \bar{c}_{0} \bar{p}_{x}\right. \\
& \left.+\bar{c}_{j} \bar{p}_{x}+\bar{d}+u^{2} / 12\right)+\left(1+2 \varepsilon^{-1}\right) \tilde{s}_{i} \delta^{2} \\
\phi_{i, 23}^{n \Psi}= & \sum_{j=1}^{n}(1-\bar{\alpha})\left(h_{j i}\right)^{2} u^{2} / 12+\left(1+2 \varepsilon^{-1}\right) \tilde{s}_{i} \delta^{2} \\
\varrho_{1}= & (1+\varepsilon) \bar{f}_{0} \bar{p}_{\pi_{i}}+\left(1+\varepsilon^{-1}\right) \bar{f}_{0}^{d} \bar{p}_{\pi_{i}} \\
& +\sum_{i=1}^{r}\left((1+\varepsilon) \bar{f}_{i}+\left(1+\varepsilon^{-1}\right) \bar{f}_{i}^{d}\right) \bar{p}_{x}+\bar{b}
\end{aligned}
$$

Remark 3 It is worth noting that the condition (13) in Theorem 3 includes all the information about plant $d y$ namics, quantizations, cyber-attacks, filtering scheme as well as communication topology. In practice, the bound of system parameters in Assumption 1, the quantizer level, the communication topology as well as the statistical characteristics of noises can all be made available to the filter design through parameter identification. Therefore, the condition (13) is essentially an algebraic polynomial inequality on $\varepsilon$ and $\bar{p}_{\pi_{i}}$. It is fairly easy, via Matlab software tools, to verify whether or not there exists a region of $\left(\varepsilon, \bar{p}_{\pi_{i}}\right)$ that guarantees (13). In addition, it can be checked if the initial condition $\Pi_{i, j \mid j}=P_{i, j \mid j} \leq \bar{p}_{\pi_{i}} I$ $(-\tau \leq j \leq 0)$ holds for some $\bar{p}_{\pi_{i}}$.

Remark 4 In this paper, the filter parameters $K_{i, k+1}^{1}$ and $K_{i, k+1}^{2}$ can be obtained in an online manner (as described in Theorem 2) in order to minimize the upper bound of filtering error covariance. Furthermore, the boundedness of the obtained sequence on the filtering error covariance is discussed in Theorem 3, which is actually concerned with the stability issues of the recursive Kalman filtering algorithms that have received particular attention in the area. As opposed to the single filter parameter in traditional stability analysis [17, 27], two filter parameters are considered in our paper that deal with, respectively, the reliable data from the node itself and the possibly unreliable data from the neighboring nodes. The introduction of the two filter parameters reflects the distributed nature of the filter dynamics but also brings in essential difficulties in the stability analysis as traditional methods (e.g. those in [17, 27]) are no longer applicable. In this paper, the mathematical induction method combined with the properties of matrix analysis is utilized to overcome the difficulties and obtain the desired sufficient conditions that are related to both the quantization and the attack. Actually, it can be seen from Theorem 3 that the solvability of (13) is dependent on both $\delta$ and $u$.

\section{Illustrative example}

In this section, we present a simulation example to illustrate the effectiveness of the proposed distributed filter 


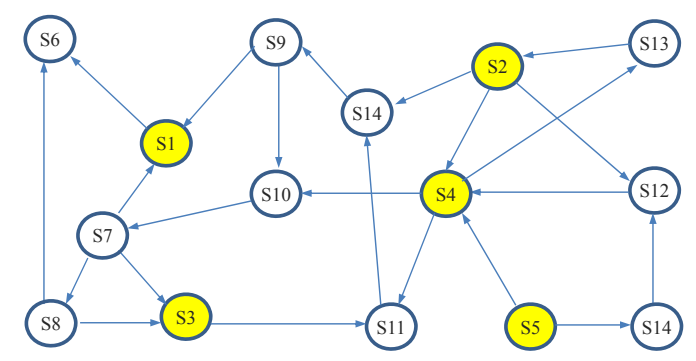

Fig. 1. Topological structure of the sensor network.

design scheme for discrete-time stochastic system with multiplicative noises through sensor networks.

The target plant considered is modeled by (1) with $r=$ $\tau=1$ and the following parameters:

$$
\begin{gathered}
A_{0, k}=\left[\begin{array}{cc}
1.0 & 0.125+0.1 \sin (0.2 k) \\
-0.2 & 0.95
\end{array}\right] \\
A_{1, k}=\left[\begin{array}{cc}
0 & 0 \\
0 & 0.1
\end{array}\right], A_{0, k}^{d}=\left[\begin{array}{cc}
-0.01 & 0 \\
0 & 0.05
\end{array}\right] \\
A_{1, k}^{d}=\left[\begin{array}{cc}
0 & 0 \\
0 & -0.01
\end{array}\right], \quad B_{k}=\left[\begin{array}{c}
0.01 \\
-0.01
\end{array}\right] \\
C_{0, k}=\left[\begin{array}{ll}
1 & 0.1
\end{array}\right], \quad C_{1, k}=\left[\begin{array}{ll}
-0.01 & 0
\end{array}\right], \quad D_{k}=0.05 .
\end{gathered}
$$

The sensor network shown in Fig. 1 has the elements in the adjacency matrix $\mathscr{H}$ are $h_{j i}=0.5$ when sensor $i$ can obtain information from sensor $j$, otherwise $h_{j i}=0$.

In this example, the probability $\bar{\alpha}$ of the cyber-attacks, the length $u$ of the quantizer level and the bound $\delta$ are, respectively, selected as $0.15,0.01$ and 0.2 . The initial conditions are set as $x_{-1}=\left[\begin{array}{lll}0.4 & 0.3\end{array}\right]^{T}, x_{0}=\left[\begin{array}{ll}2.5 & -3\end{array}\right]^{T}$, $P_{i,-1 \mid-1}=P_{i, 0 \mid 0}=I(i=1,2, \cdots, 5), X_{-1}=0.1 I$ and $X_{0}=0.2 I$. In addition, the attack signal is set to be $0.2 \sin \left(r_{i, k}\right)$ where $r_{i, k}$ obeys the Gaussian distribution $\mathcal{N}\left(0,1^{2}\right)$.

In order to compare with the centralized Kalman filtering, we consider the node S5 without neighbors, that is, we add the in-degree $\hbar_{\text {in }}^{5}=0$ Fig. 1 . On this node, the traditional Kalman filtering algorithm is utilized to obtain the estimated state and the filtering error covariance. Simulation results are shown in Figs. 2-5, where Fig. 2 plots the measurements and the actually received signals where the red broken lines show the attack phenomena. Fig. 3 depicts the trajectories for the system states (blue lines) and their estimates (red lines). In addition, we can find from Fig. 4 that the innovation information from the node itself undertakes an important role to improve the filtering performance. In Fig. 5, we aim to examine how success ratio of the launched attacks influences the filter performance. It can be observed that the trace of $\Pi_{2, k \mid k}$ at $k=100$ increases with increased $\bar{\alpha}$. Obviously, when $\bar{\alpha}>0.2$, the effect from attacks is quite limited which means that the proposed distributed filter is of the expected "robustness" against unreliable measurements resulting from multiplicative noises, uniform quantizations and intermittent deception attacks.

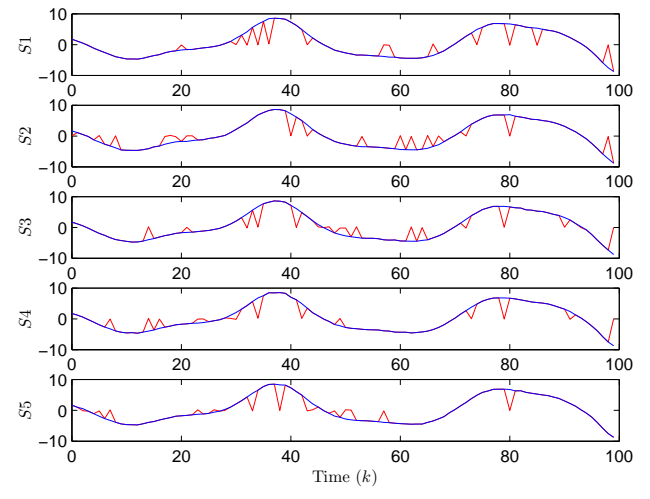

Fig. 2. The measurements and the received signals.

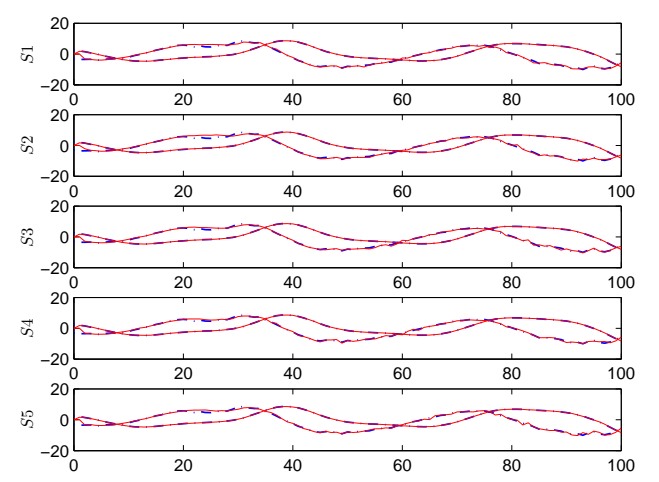

Fig. 3. The system states and their estimation (two elements for considered system state).

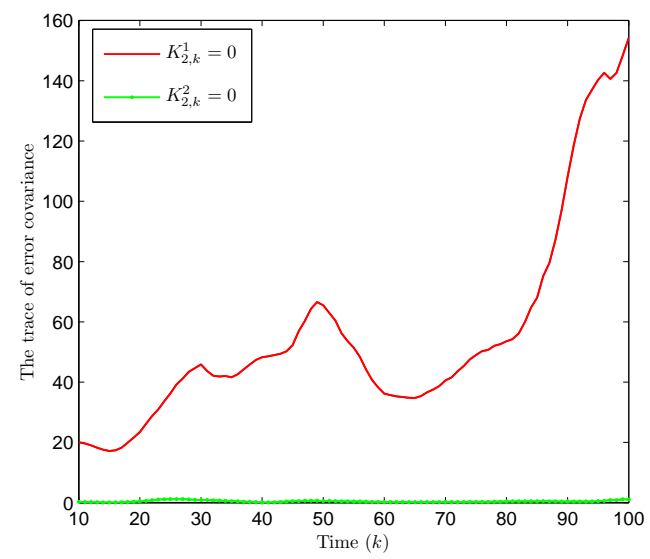

Fig. 4. The trace of filtering error covariance for different $K_{2, k}^{1}$ and $K_{2, k}^{2}$.

\section{Conclusions}

In this paper, the distributed recursive filtering problem has been investigated a class of discrete time-delayed stochastic systems subject to effects of both the uniform quantization and the deception attacks. A distributed filter has been designed that fuses the unreliable data 


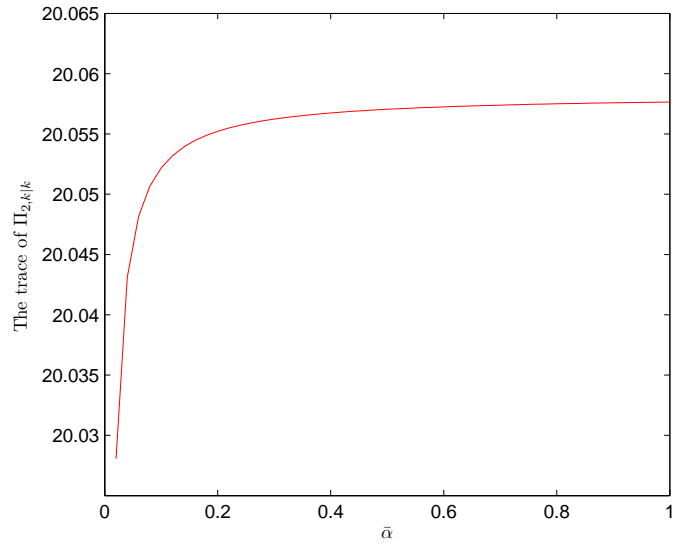

Fig. 5. The trace of $\Pi_{2, k \mid k}$ for varying $\bar{\alpha}$ on $k=100$.

corrupted by noises, quantization errors and possible deception attacks, where the filtering algorithm is shown to be efficient in reducing the computation burden resulting from time delays and the large number of sensor nodes. The complicated coupling issues between the filtering errors and observed states have been addressed in the performance analysis. An upper bound on the filtering error covariance has been guaranteed and then minimized by means of solving two Riccati-like difference equations. In addition, by utilizing the mathematical induction method, a sufficient condition has been proposed under which the filtering error covariance is bounded as time trends to infinity. Finally, an example has been provided to illustrate the effectiveness of the proposed filter approach. Further research topics would be to extend the main results of this paper to other more complex systems (e.g. [5, 10, 14,33-35]).

\section{Appendix I: Proof of Lemma 2}

Along the trajectory of system (1), it can be derived that

$$
\begin{aligned}
X_{k+1}= & B_{k} B_{k}^{T}+\sum_{s=0}^{r}\left\{A_{s, k} \mathbb{E}\left\{x_{k} x_{k}^{T}\right\} A_{s, k}^{T}\right. \\
& +A_{s, k}^{d} \mathbb{E}\left\{x_{k-\tau} x_{k-\tau}^{T}\right\}\left(A_{s, k}^{d}\right)^{T} \\
& +A_{s, k} \mathbb{E}\left\{x_{k} x_{k-\tau}^{T}\right\}\left(A_{s, k}^{d}\right)^{T} \\
& \left.+A_{s, k}^{d} \mathbb{E}\left\{x_{k-\tau} x_{k}^{T}\right\} A_{s, k}^{T}\right\} .
\end{aligned}
$$

By utilizing the element inequality $x y^{T}+y x^{T} \leq \varepsilon x x^{T}+$ $\varepsilon^{-1} y y^{T}$ for $\forall x, y \in \mathbb{R}^{n}$, one has

$$
\begin{aligned}
X_{k+1} \leq & B_{k} B_{k}^{T}+\sum_{s=0}^{r}\left\{\left(1+\varepsilon_{k}\right) A_{s, k} \mathbb{E}\left\{x_{k} x_{k}^{T}\right\} A_{s, k}^{T}\right. \\
& \left.+\left(1+\varepsilon_{k}^{-1}\right) A_{s, k}^{d} \mathbb{E}\left\{x_{k-\tau} x_{k-\tau}^{T}\right\}\left(A_{s, k}^{d}\right)^{T}\right\} \\
= & \left(1+\varepsilon_{k}\right) \sum_{s=0}^{r} A_{s, k} X_{k} A_{s, k}^{T}+B_{k} B_{k}^{T} \\
& +\left(1+\varepsilon_{k}^{-1}\right) \sum_{s=0}^{r} A_{s, k}^{d} X_{k-\tau}\left(A_{s, k}^{d}\right)^{T}
\end{aligned}
$$

which completes the proof.

\section{Appendix II: Proof of Theorem 1}

First, the covariance matrix $P_{i, k+1 \mid k}$ of one-step prediction error is given by

$$
\begin{aligned}
& P_{i, k+1 \mid k} \\
= & \mathbb{E}\left\{\left(x_{k+1}-\hat{x}_{i, k+1 \mid k}\right)\left(x_{k+1}-\hat{x}_{i, k+1 \mid k}\right)^{T}\right\} \\
\leq & \left(1+\varepsilon_{k}\right) A_{0, k} P_{i, k \mid k} A_{0, k}^{T}+B_{k} B_{k}^{T}+\left(1+\varepsilon_{k}^{-1}\right) A_{0, k}^{d} \\
& \times P_{i, k-\tau \mid k-\tau}\left(A_{0, k}^{d}\right)^{T}+\left(1+\varepsilon_{k}\right) \sum_{i=1}^{r} A_{i, k} X_{k} A_{i, k}^{T} \\
& +\left(1+\varepsilon_{k}^{-1}\right) \sum_{i=1}^{r} A_{i, k}^{d} X_{k-\tau}\left(A_{i, k}^{d}\right)^{T} .
\end{aligned}
$$

Using Lemma 1, one has $P_{i, k+1 \mid k} \leq \Pi_{i, k+1 \mid k}$. In what follows, for the purpose of simplicity, we introduce the notations:

$$
\begin{aligned}
& \mathcal{C}_{k+1}=\left[\begin{array}{lll}
\mathcal{C}_{1, k+1}^{T} & \cdots & \mathcal{C}_{n, k+1}^{T}
\end{array}\right]^{T}, \\
& \mathcal{H}_{i}=[\underbrace{0 \cdots 0}_{i-1} \quad \hbar_{i n}^{i} \underbrace{0 \cdots 0}_{n-i}], \\
& W_{k+1}=\operatorname{diag}\left\{\varpi_{1, k+1}, \cdots, \varpi_{n, k+1}\right\} \text {, } \\
& \Lambda_{k+1}^{1}=\left[\begin{array}{lll}
\alpha_{1, k+1} & \cdots & \alpha_{n, k+1}
\end{array}\right]^{T} \text {, } \\
& \Lambda_{k+1}^{2}=\left[\begin{array}{lll}
\bar{\alpha}-\alpha_{1, k+1} & \cdots & \bar{\alpha}-\alpha_{n, k+1}
\end{array}\right]^{T}, \\
& \Lambda_{k+1}^{3}=\operatorname{diag}\left\{1-\alpha_{1, k+1}, \cdots, 1-\alpha_{n, k+1}\right\} \text {. }
\end{aligned}
$$

Subtracting (5b) from (1) leads to

$$
\begin{aligned}
& e_{i, k+1 \mid k+1} \\
= & x_{k+1}-\hat{x}_{i, k+1 \mid k+1} \\
= & \left(I-\hbar_{i n}^{i} K_{i, k+1}^{1} C_{0, k+1}-\sum_{j \in \mathcal{N}_{i}}(1-\bar{\alpha}) h_{j i} K_{i, k+1}^{2}\right. \\
& \left.\times C_{0, k+1}\right) e_{i, k+1 \mid k}-\hbar_{i n}^{i} K_{i, k+1}^{1}\left(\varpi_{i, k+1} C_{i, k+1} x_{k+1}\right. \\
& \left.+D_{k+1} v_{i, k+1}+q_{i, k+1}\right)-K_{i, k+1}^{2} \sum_{j \in \mathcal{N}_{i}} h_{j i}((\bar{\alpha} \\
& \left.\left.-\alpha_{j, k+1}\right) C_{0, k+1}+\left(1-\alpha_{j, k+1}\right) \varpi_{j, k+1} C_{j, k+1}\right) x_{k+1} \\
& -K_{i, k+1}^{2} \sum_{j \in \mathcal{N}_{i}} h_{j i}\left(\alpha_{j, k+1} \xi_{k+1}+\left(1-\alpha_{j, k+1}\right) q_{j, k+1}\right) \\
& -K_{i, k+1}^{2} \sum_{j \in \mathcal{N}_{i}}\left(1-\alpha_{j, k+1}\right) h_{j i} D_{k+1} v_{j, k+1} \\
= & \Psi_{i, k+1}^{0} e_{i, k+1 \mid k}-K_{i, k+1}^{1}\left(\mathcal{H}_{i} \otimes I\right)\left(W_{k+1} \otimes I\right) \mathcal{C}_{k+1} x_{k+1} \\
& -K_{i, k+1}^{1}\left(\mathcal{H}_{i} \otimes I\right)\left(I \otimes D_{k+1}\right) v_{k+1}-K_{i, k+1}^{1}\left(\mathcal{H}_{i}\right. \\
& \otimes I) q_{k+1}-K_{i, k+1}^{2}\left(\overline{\mathcal{H}}_{i} \otimes I\right)\left(\Lambda_{k+1}^{2} \otimes I\right) C_{0, k+1} x_{k+1} \\
& -K_{i, k+1}^{2}\left(\overline{\mathcal{H}}_{i} \otimes I\right)\left(\Lambda_{k+1}^{3} W_{k+1} \otimes I\right) \mathcal{C}_{k+1} x_{k+1} \\
& -K_{i, k+1}^{2}\left(\overline{\mathcal{H}}_{i} \otimes I\right)\left(\Lambda_{k+1}^{1} \otimes I\right) \xi_{k+1} \\
& -K_{i, k+1}^{2}\left(\overline{\mathcal{H}}_{i} \otimes I\right)\left(\Lambda_{k+1}^{3} \otimes I\right) q_{k+1} \\
& -K_{i, k+1}^{2}\left(\overline{\mathcal{H}}_{i} \otimes I\right)\left(\Lambda_{k+1}^{3} \otimes I\right)\left(I \otimes D_{k+1}\right) v_{k+1} \\
&
\end{aligned}
$$


with

$$
\overline{\mathcal{H}}_{i}=\left[\hbar_{j}^{i}\right]_{1 \times n}= \begin{cases}\hbar_{j}^{i}=h_{j i}, & j \in \mathcal{N}_{i}, \\ \hbar_{j}^{i}=0, & j \notin \mathcal{N}_{i} .\end{cases}
$$

Now, let us calculate the filtering error covariance $P_{i, k+1 \mid k+1}$ as follows:

$P_{i, k+1 \mid k+1}$

$=\mathbb{E}\left\{\left(x_{k+1}-\hat{x}_{i, k+1 \mid k+1}\right)\left(x_{k+1}-\hat{x}_{i, k+1 \mid k+1}\right)^{T}\right\}$

$=\Psi_{i, k+1}^{0} \mathbb{E}\left\{e_{i, k+1 \mid k} e_{i, k+1 \mid k}^{T}\right\} \Psi_{i, k+1}^{0 T}$

- $\Psi_{i, k+1}^{0} \mathbb{E}\left\{e_{i, k+1 \mid k} \xi_{k+1}^{T}\left(\Lambda_{k+1}^{1} \otimes I\right)^{T}\right\}\left(\overline{\mathcal{H}}_{i} \otimes I\right)^{T} K_{i, k+1}^{2 T}$

$-K_{i, k+1}^{2}\left(\mathcal{S}_{i} \otimes I\right) \mathbb{E}\left\{\left(\Lambda_{k+1}^{1} \otimes I\right) \xi_{k+1} e_{i, k+1 \mid k}^{T}\right\} \Psi_{i, k+1}^{0 T}$

$+K_{i, k+1}^{1}\left(\mathcal{H}_{i} \otimes I\right) \mathbb{E}\left\{\left(W_{k+1} \otimes I\right) \mathcal{C}_{k+1} x_{k+1}\right.$

$\left.\times x_{k+1}^{T} \mathcal{C}_{k+1}^{T}\left(W_{k+1} \otimes I\right)^{T}\right\}\left(\mathcal{H}_{i} \otimes I\right)^{T} K_{i, k+1}^{1 T}$

$+K_{i, k+1}^{1}\left(\mathcal{H}_{i} \otimes I\right)\left(I \otimes D_{k+1}\right) \mathbb{E}\left\{v_{k+1}\right.$

$\left.\times v_{k+1}^{T}\right\}\left(I \otimes D_{k+1}\right)^{T}\left(\mathcal{H}_{i} \otimes I\right)^{T} K_{i, k+1}^{1 T}$

$+K_{i, k+1}^{1}\left(\mathcal{H}_{i} \otimes I\right) \mathbb{E}\left\{q_{k+1} q_{k+1}^{T}\right\}\left(\mathcal{H}_{i} \otimes I\right)^{T} K_{i, k+1}^{1 T}$

$+K_{i, k+1}^{2}\left(\overline{\mathcal{H}}_{i} \otimes I\right) \mathbb{E}\left\{\left(\Lambda_{k+1}^{2} \otimes I\right) C_{0, k+1} x_{k+1}\right.$

$\left.\times x_{k+1}^{T} C_{0, k+1}^{T}\left(\Lambda_{k+1}^{2} \otimes I\right)^{T}\right\}\left(\overline{\mathcal{H}}_{i} \otimes I\right)^{T} K_{i, k+1}^{2 T}$

$-K_{i, k+1}^{2}\left(\overline{\mathcal{H}}_{i} \otimes I\right) \mathbb{E}\left\{\left(\Lambda_{k+1}^{2} \otimes I\right) C_{0, k+1} x_{k+1}\right.$

$\left.\times \xi_{k+1}^{T}\left(\Lambda_{k+1}^{1} \otimes I\right)^{T}\right\}\left(\overline{\mathcal{H}}_{i} \otimes I\right)^{T} K_{i, k+1}^{2 T}$

$-K_{i, k+1}^{2}\left(\overline{\mathcal{H}}_{i} \otimes I\right) \mathbb{E}\left\{\left(\Lambda_{k+1}^{1} \otimes I\right) \xi_{k+1}\right.$

$\left.\times x_{k+1}^{T} C_{0, k+1}^{T}\left(\Lambda_{k+1}^{2} \otimes I\right)^{T}\right\}\left(\overline{\mathcal{H}}_{i} \otimes I\right)^{T} K_{i, k+1}^{2 T}$

$+K_{i, k+1}^{2}\left(\overline{\mathcal{H}}_{i} \otimes I\right) \mathbb{E}\left\{\left(\Lambda_{k+1}^{3} W_{k+1} \otimes I\right) \mathcal{C}_{k+1} x_{k+1}\right.$

$\left.\times x_{k+1}^{T} \mathcal{C}_{k+1}^{T}\left(\Lambda_{k+1}^{3} W_{k+1} \otimes I\right)^{T}\right\}\left(\overline{\mathcal{H}}_{i} \otimes I\right)^{T} K_{i, k+1}^{2 T}$

$+K_{i, k+1}^{2}\left(\overline{\mathcal{H}}_{i} \otimes I\right) \mathbb{E}\left\{\left(\Lambda_{k+1}^{1} \otimes I\right) \xi_{k+1}\right.$

$\left.\times \xi_{k+1}^{T}\left(\Lambda_{k+1}^{1} \otimes I\right)^{T}\right\}\left(\overline{\mathcal{H}}_{i} \otimes I\right)^{T} K_{i, k+1}^{2 T}$

$+K_{i, k+1}^{2}\left(\overline{\mathcal{H}}_{i} \otimes I\right) \mathbb{E}\left\{\left(\Lambda_{k+1}^{3} \otimes I\right)\left(I \otimes D_{k+1}\right) v_{k+1}\right.$

$\left.\times v_{k+1}^{T}\left(I \otimes D_{k+1}\right)^{T}\left(\Lambda_{k+1}^{3} \otimes I\right)^{T}\right\}\left(\overline{\mathcal{H}}_{i} \otimes I\right)^{T} K_{i, k+1}^{2 T}$

$+K_{i, k+1}^{2}\left(\overline{\mathcal{H}}_{i} \otimes I\right) \mathbb{E}\left\{\left(\Lambda_{k+1}^{3} \otimes I\right) q_{k+1}\right.$

$\left.\times q_{k+1}^{T}\left(\Lambda_{k+1}^{3} \otimes I\right)^{T}\right\}\left(\overline{\mathcal{H}}_{i} \otimes I\right)^{T} K_{i, k+1}^{2 T}$

$\leq\left(1+\varepsilon_{k}\right) \Psi_{i, k+1}^{0} P_{i, k+1 \mid k} \Psi_{i, k+1}^{0 T}$

$+K_{i, k+1}^{1}\left(\mathcal{H}_{i} \otimes I\right) X_{k+1}^{C}\left(\mathcal{H}_{i} \otimes I\right)^{T} K_{i, k+1}^{1 T}$

$+K_{i, k+1}^{1}\left(\mathcal{H}_{i} \mathcal{H}_{i}^{T} \otimes D_{k+1} D_{k+1}^{T}\right) K_{i, k+1}^{1 T}$

$+\frac{u^{2}}{12} K_{i, k+1}^{1}\left(\mathcal{H}_{i} \mathcal{H}_{i}^{T} \otimes I\right) K_{i, k+1}^{1 T}$

$+\left(1+\varepsilon_{k}\right) \bar{\alpha}(1-\bar{\alpha}) K_{i, k+1}^{2}\left(\overline{\mathcal{H}}_{i} \overline{\mathcal{H}}_{i}^{T}\right.$

$\left.\otimes C_{0, k+1} X_{k+1} C_{0, k+1}^{T}\right) K_{i, k+1}^{2 T}$

$+(1-\bar{\alpha}) K_{i, k+1}^{2}\left(\overline{\mathcal{H}}_{i} \otimes I\right) X_{k+1}^{C}\left(\overline{\mathcal{H}}_{i} \otimes I\right)^{T} K_{i, k+1}^{2 T}$

$+\left(1+2 \varepsilon_{k}^{-1}\right) \delta^{2} K_{i, k+1}^{2}\left(\overline{\mathcal{H}}_{i} \otimes I\right)\left(\bar{\alpha}^{2}\left(\mathbf{1 1}^{T}\right) \otimes I\right.$

$\left.+\left(\bar{\alpha}-\bar{\alpha}^{2}\right) I\right)\left(\overline{\mathcal{H}}_{i} \otimes I\right)^{T} K_{i, k+1}^{2 T}$

$+(1-\bar{\alpha}) K_{i, k+1}^{2}\left(\overline{\mathcal{H}}_{i} \overline{\mathcal{H}}_{i}^{T} \otimes D_{k+1} D_{k+1}^{T}\right) K_{i, k+1}^{2 T}$

$+\frac{(1-\bar{\alpha}) u^{2}}{12} K_{i, k+1}^{2}\left(\overline{\mathcal{H}}_{i} \overline{\mathcal{H}}_{i}^{T} \otimes I\right) K_{i, k+1}^{2 T}$

Using the properties of Kronecker product to the above matrix inequality results in

$$
\begin{aligned}
& P_{i, k+1 \mid k+1} \leq \Psi_{i, k+1}^{0} P_{i, k+1 \mid k} \Psi_{i, k+1}^{0 T}+K_{i, k+1}^{1} \\
& \quad \times \Psi_{i, k+1}^{1} K_{i, k+1}^{1 T}+K_{i, k+1}^{2} \Psi_{i, k+1}^{23} K_{i, k+1}^{2 T}
\end{aligned}
$$

which implies that the second inequality in (10) is true.

\section{Appendix III: Proof of Theorem 2}

According to Theorem 1, the design of gains $K_{k+1}^{1}$ and $K_{k+1}^{2}$ needs to minimize Trace $\left(\Pi_{i, k+1 \mid k+1}\right)$. For this purpose, taking the partial derivative of Trace $\left(\Pi_{i, k+1 \mid k+1}\right)$ with respect to $K_{k+1}^{1}$ and $K_{k+1}^{2}$, and letting the derivative be zero, one has

$$
\begin{aligned}
& \frac{\partial \operatorname{Trace}\left(\Pi_{i, k+1 \mid k+1}\right)}{\partial K_{i, k+1}^{1}}=0 \\
\Rightarrow & -\hbar_{i n}^{i} \Psi_{i, k+1}^{0} \Pi_{i, k+1 \mid k} C_{0, k+1}^{T}+K_{i, k+1}^{1} \Psi_{i, k+1}^{1}=0 . \\
& \frac{\partial \operatorname{Trace}\left(\Pi_{i, k+1 \mid k+1}\right)}{\partial K_{i, k+1}^{2}}=0 \\
\Rightarrow & -\hbar_{i n}^{i}(1-\bar{\alpha}) \Psi_{i, k+1}^{0} \Pi_{i, k+1 \mid k} C_{0, k+1}^{T}+K_{i, k+1}^{2} \Psi_{i, k+1}^{23}=0 .
\end{aligned}
$$

Subsequently, we have

$$
\begin{aligned}
& -\hbar_{i n}^{i}\left(I-\hbar_{i n}^{i}(1-\bar{\alpha}) K_{i, k+1}^{2} C_{0, k+1}\right) \Pi_{i, k+1 \mid k} C_{0, k+1}^{T} \\
& \quad+K_{i, k+1}^{1}\left(\left(\hbar_{i n}^{i}\right)^{2} C_{0, k+1} \Pi_{i, k+1 \mid k} C_{0, k+1}^{T}+\Psi_{i, k+1}^{1}\right)=0 \\
& -\hbar_{i n}^{i}(1-\bar{\alpha})\left(I-\hbar_{i n}^{i} K_{i, k+1}^{1} C_{0, k+1}\right) \Pi_{i, k+1 \mid k} C_{0, k+1}^{T} \\
& \quad+K_{i, k+1}^{2}\left(\left(\hbar_{i n}^{i}\right)^{2}(1-\bar{\alpha})^{2} C_{0, k+1}\right. \\
& \left.\quad \times \Pi_{i, k+1 \mid k} C_{0, k+1}^{T}+\Psi_{i, k+1}^{23}\right)=0
\end{aligned}
$$

which can be further simplified as follows:

$$
\begin{aligned}
K_{i, k+1}^{1} \mathcal{S}_{i, k+1}^{1} & +(1-\bar{\alpha})\left(\hbar_{i n}^{i}\right)^{2} K_{i, k+1}^{2} \mathcal{S}_{i, k+1}^{0} \\
& -\hbar_{i n}^{i} \Pi_{i, k+1 \mid k} C_{0, k+1}^{T}=0 \\
K_{i, k+1}^{2} \mathcal{S}_{i, k+1}^{2} & +(1-\bar{\alpha})\left(\hbar_{i n}^{i}\right)^{2} K_{i, k+1}^{1} \mathcal{S}_{i, k+1}^{0} \\
& -(1-\bar{\alpha}) \hbar_{i n}^{i} \Pi_{i, k+1 \mid k} C_{0, k+1}^{T}=0 .
\end{aligned}
$$

Furthermore, the following

$$
\begin{aligned}
\Omega_{i, k+1}^{1}= & \mathcal{S}_{i, k+1}^{1}-(1-\bar{\alpha})^{2}\left(\hbar_{i n}^{i}\right)^{4} \mathcal{S}_{i, k+1}^{0}\left(\mathcal{S}_{i, k+1}^{2}\right)^{-1} \mathcal{S}_{i, k+1}^{0} \\
= & \Psi_{i, k+1}^{1}+\left(\hbar_{i n}^{i}\right)^{2}\left[\mathcal{S}_{i, k+1}^{0}-\left((1-\bar{\alpha})^{2}\left(\hbar_{i n}^{i}\right)^{2} \mathcal{S}_{i, k+1}^{0}\right.\right. \\
& \left.\left.+\Psi_{i, k+1}^{23}-\Psi_{i, k+1}^{23}\right)\left(\mathcal{S}_{i, k+1}^{2}\right)^{-1} \mathcal{S}_{i, k+1}^{0}\right] \\
= & \Psi_{i, k+1}^{1}+\left(\hbar_{i n}^{i}\right)^{2} \Psi_{i, k+1}^{23}\left(\mathcal{S}_{i, k+1}^{2}\right)^{-1} \mathcal{S}_{i, k+1}^{0} \\
= & \Psi_{i, k+1}^{1}+(1-\bar{\alpha})^{-2} \\
& \times\left(\Psi_{i, k+1}^{23}-\Psi_{i, k+1}^{23}\left(\mathcal{S}_{i, k+1}^{2}\right)^{-1} \Psi_{i, k+1}^{23}\right) \\
> & \Psi_{i, k+1}^{1}
\end{aligned}
$$




$$
\begin{aligned}
\Omega_{i, k+1}^{2}= & \mathcal{S}_{i, k+1}^{2}-(1-\bar{\alpha})^{2}\left(\hbar_{i n}^{i}\right)^{4} \mathcal{S}_{i, k+1}^{0}\left(\mathcal{S}_{i, k+1}^{1}\right)^{-1} \mathcal{S}_{i, k+1}^{0} \\
= & \Psi_{i, k+1}^{23}+(1-\bar{\alpha})^{2} \\
& \times\left(\Psi_{i, k+1}^{1}-\Psi_{i, k+1}^{1}\left(\mathcal{S}_{i, k+1}^{1}\right)^{-1} \Psi_{i, k+1}^{1}\right) \\
> & \Psi_{i, k+1}^{23}
\end{aligned}
$$

are true. Therefore, taking (20)-(22) into consideration, we can obtain the desired filter gain matrices. Furthermore, the upper bound for the filtering error covariance $\Pi_{i, k+1 \mid k+1}$ is recursively calculated by Riccati-like difference equation (11).

\section{Appendix IV: Proof of Theorem 3}

In order to prove the boundedness, the mathematical induction method is utilized to deal with the difficulty coming from both two filtering gains and the coupling with plant states. For this purpose, suppose that $\underline{p}_{\pi_{i}} I \leq$ $\Pi_{i, k \mid k} \leq \bar{p}_{\pi_{i}} I$ holds and then show that it is also true at the inductive step (that is, at $k+1$ instants).

For the sake of simplicity, we fix $\varepsilon_{k}=\varepsilon$ for any $k$, and can obtain from (11) that

$$
\begin{aligned}
& \Pi_{i, k+1 \mid k} \\
= & (1+\varepsilon) A_{0, k} \Pi_{i, k \mid k} A_{0, k}^{T} \\
& +\left(1+\varepsilon^{-1}\right) A_{0, k}^{d} \Pi_{i, k-\tau \mid k-\tau}\left(A_{0, k}^{d}\right)^{T} \\
& +(1+\varepsilon) \sum_{i=1}^{r} A_{i, k} X_{k} A_{i, k}^{T} \\
& +\left(1+\varepsilon^{-1}\right) \sum_{i=1}^{r} A_{i, k}^{d} X_{k-\tau}\left(A_{i, k}^{d}\right)^{T}+B_{k} B_{k}^{T} \\
\leq & \varrho_{1} I
\end{aligned}
$$

In what follows, it is not difficult to see

$$
\begin{aligned}
& \Psi_{i, k+1}^{0} \Pi_{i, k+1 \mid k} \Psi_{i, k+1}^{0 T} \\
= & \left(I-\hbar_{i n}^{i} K_{i, k+1}^{1} C_{0, k+1}-\hbar_{i n}^{i}(1-\bar{\alpha}) K_{i, k+1}^{2} C_{0, k+1}\right) \\
& \times \Pi_{i, k+1 \mid k}\left(I-\hbar_{i n}^{i} K_{i, k+1}^{1} C_{0, k+1}\right. \\
& \left.-\hbar_{i n}^{i}(1-\bar{\alpha}) K_{i, k+1}^{2} C_{0, k+1}\right)^{T} \\
\leq & 3 \Pi_{i, k+1 \mid k}+3\left(\hbar_{i n}^{i}\right)^{2} K_{i, k+1}^{1} \mathcal{S}_{i, k+1}^{0}\left(K_{i, k+1}^{1}\right)^{T} \\
& +3\left(\hbar_{i n}^{i}\right)^{2}(1-\bar{\alpha})^{2} K_{i, k+1}^{2} \mathcal{S}_{i, k+1}^{0}\left(K_{i, k+1}^{2}\right)^{T}
\end{aligned}
$$

Substituting (24) into (11) leads to

$$
\begin{aligned}
& \Pi_{i, k+1 \mid k+1} \\
\leq & 3 \Pi_{i, k+1 \mid k}+K_{i, k+1}^{1} \Psi_{i, k+1}^{1}\left(K_{i, k+1}^{1}\right)^{T} \\
& +K_{i, k+1}^{2} \Psi_{i, k+1}^{23}\left(K_{i, k+1}^{2}\right)^{T} \\
& +3\left(\hbar_{i n}^{i}\right)^{2} K_{i, k+1}^{1} \mathcal{S}_{i, k+1}^{0}\left(K_{i, k+1}^{1}\right)^{T} \\
& +3\left(\hbar_{i n}^{i}\right)^{2}(1-\bar{\alpha})^{2} K_{i, k+1}^{2} \mathcal{S}_{i, k+1}^{0}\left(K_{i, k+1}^{2}\right)^{T}
\end{aligned}
$$

where $\Psi_{i, k+1}^{23}=\Psi_{i, k+1}^{2}+\Psi_{i, k+1}^{3}$.

On the other hand, reviewing (21)-(22) and using the property of positive definite matrices, one has

$$
\begin{aligned}
\left(\Omega_{i, k+1}^{1}\right)^{-1} & \mathcal{S}_{i, k+1}^{0}\left(\Omega_{i, k+1}^{1}\right)^{-1} \\
& \leq \lambda_{\max }\left(\mathcal{S}_{i, k+1}^{0}\right)\left(\Omega_{i, k+1}^{1} \Omega_{i, k+1}^{1}\right)^{-1} \\
& \leq \lambda_{\max }\left(\mathcal{S}_{i, k+1}^{0}\right) \lambda_{1}^{2}\left(\left(\Psi_{i, k+1}^{1}\right)^{-1}\right) I, \\
\left(\Omega_{i, k+1}^{2}\right)^{-1} & \mathcal{S}_{i, k+1}^{0}\left(\Omega_{i, k+1}^{2}\right)^{-1} \\
& \leq \lambda_{\max }\left(\mathcal{S}_{i, k+1}^{0}\right)\left(\Omega_{i, k+1}^{2} \Omega_{i, k+1}^{2}\right)^{-1} \\
& \leq \lambda_{\max }\left(\mathcal{S}_{i, k+1}^{0}\right) \lambda_{1}^{2}\left(\left(\Psi_{i, k+1}^{23}\right)^{-1}\right) I
\end{aligned}
$$

Then, denoting

$$
\Lambda_{i, k+1}=\left(\hbar_{i n}^{i}\right)^{2} \mathcal{S}_{i, k+1}^{0}-(1-\bar{\alpha})^{2}\left(\hbar_{i n}^{i}\right)^{4} \mathcal{S}_{i, k+1}^{0}\left(\mathcal{S}_{i, k+1}^{2}\right)^{-1} \mathcal{S}_{i, k+1}^{0}
$$

we can check

$$
\begin{aligned}
& \Lambda_{i, k+1} \\
= & \left(\hbar_{i n}^{i}\right)^{2} \mathcal{S}_{i, k+1}^{0}-\left(\hbar_{i n}^{i}\right)^{2}\left(\mathcal{S}_{i, k+1}^{2}\right. \\
& \left.-\Psi_{i, k+1}^{23}\right)\left(\mathcal{S}_{i, k+1}^{2}\right)^{-1} \mathcal{S}_{i, k+1}^{0} \\
= & \left(\hbar_{i n}^{i}\right)^{2} \Psi_{i, k+1}^{23}\left(\mathcal{S}_{i, k+1}^{2}\right)^{-1} \mathcal{S}_{i, k+1}^{0} \\
= & (1-\bar{\alpha})^{2} \Psi_{i, k+1}^{23}\left(\mathcal{S}_{i, k+1}^{2}\right)^{-1}\left(\mathcal{S}_{i, k+1}^{2}-\Psi_{i, k+1}^{23}\right) \\
= & \left.(1-\bar{\alpha})^{2}\left(\Psi_{i, k+1}^{23}-\Psi_{i, k+1}^{23}\left(\mathcal{S}_{i, k+1}^{2}\right)^{-1} \Psi_{i, k+1}^{23}\right)\right) \\
\geq & 0
\end{aligned}
$$

According to the above inequality, it can be derived that

$$
\begin{aligned}
& \left(\Omega_{i, k+1}^{1}\right)^{-1} \Psi_{i, k+1}^{1}\left(\Omega_{i, k+1}^{1}\right)^{-1} \\
= & \left(\Psi_{i, k+1}^{1}+\Lambda_{i, k+1}\right)^{-1} \Psi_{i, k+1}^{1}\left(\Psi_{i, k+1}^{1}+\Lambda_{i, k+1}\right)^{-1} \\
= & \left(\Psi_{i, k+1}^{1}+2 \Lambda_{i, k+1}+\Lambda_{i, k+1}\left(\Psi_{i, k+1}^{1}\right)^{-1} \Lambda_{i, k+1}\right)^{-1} \\
\leq & \left(\Psi_{i, k+1}^{1}\right)^{-1}
\end{aligned}
$$

Furthermore, using the same line of the above inequality, we have

$$
\left(\Omega_{i, k+1}^{2}\right)^{-1} \Psi_{i, k+1}^{23}\left(\Omega_{i, k+1}^{2}\right)^{-1} \leq\left(\Psi_{i, k+1}^{23}\right)^{-1}
$$

In addition, we can calculate

$$
\begin{aligned}
& \left(I-\left(\hbar_{i n}^{i}\right)^{2}(1-\bar{\alpha})^{2}\left(\mathcal{S}_{i, k+1}^{2}\right)^{-1} \mathcal{S}_{i, k+1}^{0}\right) \\
& \times\left(I-\left(\hbar_{i n}^{i}\right)^{2}(1-\bar{\alpha})^{2}\left(\mathcal{S}_{i, k+1}^{2}\right)^{-1} \mathcal{S}_{i, k+1}^{0}\right)^{T} \\
= & I-\left(\hbar_{i n}^{i}\right)^{2}(1-\bar{\alpha})^{2}\left(\mathcal{S}_{i, k+1}^{2}\right)^{-1} \mathcal{S}_{i, k+1}^{0} \\
& -\left(\hbar_{i n}^{i}\right)^{2}(1-\bar{\alpha})^{2} \mathcal{S}_{i, k+1}^{0}\left(\mathcal{S}_{i, k+1}^{2}\right)^{-1} \\
& +\left(\hbar_{i n}^{i}\right)^{4}(1-\bar{\alpha})^{4}\left(\mathcal{S}_{i, k+1}^{2}\right)^{-1} \mathcal{S}_{i, k+1}^{0} \mathcal{S}_{i, k+1}^{0}\left(\mathcal{S}_{i, k+1}^{2}\right)^{-1} \\
= & \left(\mathcal{S}_{i, k+1}^{2}\right)^{-1} \Psi_{i, k+1}^{23}+\Psi_{i, k+1}^{23}\left(\mathcal{S}_{i, k+1}^{2}\right)^{-1}-I \\
& +\left(\hbar_{i n}^{i}\right)^{4}(1-\bar{\alpha})^{4}\left(\mathcal{S}_{i, k+1}^{2}\right)^{-1} \mathcal{S}_{i, k+1}^{0} \mathcal{S}_{i, k+1}^{0}\left(\mathcal{S}_{i, k+1}^{2}\right)^{-1} \\
= & \left(\mathcal{S}_{i, k+1}^{2}\right)^{-1} \Psi_{i, k+1}^{23} \Psi_{i, k+1}^{23}\left(\mathcal{S}_{i, k+1}^{2}\right)^{-1} \\
\leq & \frac{\left(\phi_{i, 23}^{m \Psi}\right)^{2}}{\left(\phi_{i, 23}^{n \Psi}\right)^{2}} I
\end{aligned}
$$

Similarly, it can be obtained

$$
\begin{aligned}
& \left(I-\left(\hbar_{i n}^{i}\right)^{2}\left(\mathcal{S}_{i, k+1}^{1}\right)^{-1} \mathcal{S}_{i, k+1}^{0}\right) \\
& \times\left(I-\left(\hbar_{i n}^{i}\right)^{2}\left(\mathcal{S}_{i, k+1}^{1}\right)^{-1} \mathcal{S}_{i, k+1}^{0}\right)^{T} \leq \frac{\left(\phi_{i, 1}^{m \Psi}\right)^{2}}{\left(\phi_{i, 1}^{n \Psi}\right)^{2}} I
\end{aligned}
$$


Finally, in light of Lemma 3 and Lemma 4, substituting (26), (28), (29), (30) and (31) into (25) results in

$$
\Pi_{i, k+1 \mid k+1} \leq\left(3+\xi_{0}\right) \Pi_{i, k+1 \mid k} \leq\left(3+\xi_{0}\right) \varrho_{1} I \leq \bar{p}_{\pi_{i}} I
$$

Therefore, by the induction, it can be concluded that $\Pi_{i, k \mid k}<\bar{p}_{\pi_{i}} I$ is true for any $k \geq 1$, which completes the proof.

\section{References}

[1] S. Amin, G. A. Schwartz, S. Shankar Sastry, Security of interdependent and identical networked control systems, Automatica, vol. 49, no. 1, pp. 186-192, Jan. 2013.

[2] M. Basin, A. Alcorta-Garcia, and J. Rodriguez-Gonzalez, Optimal filtering for linear systems with state and observation delays, International Journal of Robust and Nonlinear Control, vol. 15, no. 17, pp. 859-871, Nov. 2005.

[3] M. Basin and R. Martinez-Zuniga, Optimal linear filtering over observations with multiple delays, International Journal of Robust and Nonlinear Control, vol. 14, no. 8, pp. 685-696, May 2004.

[4] R. W. Brockett and D. Liberzon, Quantized feedback stabilization of linear systems, IEEE Transactions on Automatic control, vol. 55, no. 7, pp. 1279-1289, Jul. 2000.

[5] H. Chen, J. Liang and Z. Wang, Pinning controllability of autonomous Boolean control networks, Science China Information Sciences, vol. 59, no. 7, Art. No. 070107, DOI: 10.1007/s11432-016-5579-8, 2016.

[6] D. Ding, G. Wei, S. Zhang, Y. Liu, and F. E. Alsaadi, On scheduling of deception attacks for discrete-time networked systems equipped with attack detectors, Neurocomputing. DOI: 10.1016/j.neucom.2016.09.009.

[7] D. Ding, Z. Wang, D. W. C. Ho, and G. Wei, Observer-based event-triggering consensus control for multi-agent systems with lossy sensors and cyber attacks, IEEE Transactions on Cybernetics. DOI: 10.1109/TCYB.2016.2582802.

[8] H. Dong, Z. Wang and H. Gao, Distributed $H_{\infty}$ filtering for a class of Markovian jump nonlinear time-delay systems over lossy sensor networks, IEEE Transactions on Industrial Electronics, vol. 60, no. 10, pp. 4665-4672, Oct. 2013

[9] H. Dong, Z. Wang, F. E. Alsaadi, and B. Ahmad, Eventtriggered robust distributed state estimation for sensor networks with state-dependent noises, International Journal of General Systems, vol. 44, no. 2, pp. 254-266, Feb. 2015.

[10] H. Dong, Z. Wang, B. Shen and D. Ding, Varianceconstrained $H_{\infty}$ control for a class of nonlinear stochastic discrete time-varying systems: the event-triggered design, Automatica, vol. 72, pp. 28-36, 2016.

[11] H. Fawzi, P. Tabuada, and S. Diggavi, Secure estimation and control for cyber-physical systems under adversarial attacks, IEEE Transactions on Automatic control, vol. 59, no. 6, pp. 1454-1467, Jun. 2014.

[12] M. A. Gandhi and L. Mili, Robust Kalman filter based on a generalized maximum-likelihood-type estimator, IEEE Transactions on Automatic control, vol. 58, no. 5, pp. 25092520, May 2010.

[13] E. Gershon, U. Shaked and I. Yaesh, $\mathcal{H}_{\infty}$ control and filtering of discrete-time stochastic systems with multiplicative noise, Automatica, Vol. 37, No. 3, pp. 409-417, Mar. 2001.

[14] N. Hou, H. Dong, Z. Wang, W. Ren and F. E. Alsaadi, Non-fragile state estimation for discrete Markovian jumping neural networks, Neurocomputing, vol. 179, pp. 238-245, 2016.

[15] J. Hu, D. Chen and J. Du, State estimation for a class of discrete nonlinear systems with randomly occurring uncertainties and distributed sensor delays, International Journal of General Systems, vol. 43, nos. 3-4, pp. 387-401, 2014.
[16] J. Hu, S. Liu, D. Ji, and S. Li, On co-design of filter and fault estimator against randomly occurring nonlinearities and randomly occurring deception attacks, International Journal of General Systems, vol. 45, no. 5, pp. 619-632, 2016.

[17] S. Kluge, K. Reif and M. Brokate, Stochastic stability of the extended Kalman filter with intermittent observations, IEEE Transactions on Automatic control, vol. 55, no. 2, pp. 514518, Feb. 2010.

[18] S. Kong, M. Saif, and H. Zhang, Optimal filtering forstochastic continuous-time systems with multiple delayed measurements, IEEE Transactions on Automatic Control, vol. 58, no. 7, pp. 1872-1877, 2013.

[19] Q. Li, B. Shen, Y. Liu, F. E. Alsaadi, Event-triggered $\mathcal{H}_{\infty}$ state estimation for discrete-time stochastic genetic regulatory networks with Markovian jumping parameters and time-varying delays, Neurocomputing, vol. 174, 912-920, Jan. 2016.

[20] Q. Li, B. Shen, J. Liang, and H. Shu, Event-triggered synchronization control for complex networks with uncertain inner coupling, International Journal of General Systems, vol. 44, no. 2, Jan. 2015, pp. 212-225.

[21] M. Long, C.-H. Wu and J. Y. Hung, Denial of service attacks on network-based control systems: impact and mitigation, IEEE Transactions on Industrial Informatics, vol. 1, no. 2, pp. 85-96, May 2005.

[22] X. Lu, L. Xie, H. Zhang and W. Wang, Robust Kalman filtering for discrete-time systems with measurement delay, IEEE Transactions on Circuits and Systems-II, vol. 54, no. 6 , pp. 522-526, Jun. 2007.

[23] L.-Z. Lu, Some new bounds for singular values and eigenvalues of matrix products, Annals of Operations Research, vol. 98, nos. 1-4, pp. 141-148, Dec. 2000.

[24] S. Marano, V. Matta, and L. Tong, Distributed detection in the presence of Byzantine attacks, IEEE Transactions on Singnal Processing, vol. 57, no. 1, pp. 16-29, Jan. 2009.

[25] R. Olfati-Saber, Distributed Kalman filtering for sensor networks, In: Proceedings of the 46th IEEE Conference on Decision and Control, New Orleans, LA, pp. 5492-5498, 2007.

[26] Z.-H. Pang and G.-P. Liu, Design and implementation of secure networked predictive control systems under deception attacks, IEEE Transactions on Control Systems Technology, vol. 20, no. 5, pp. 1334-1342, Sep. 2012.

[27] K. Reif, S. Günther, E. Yaz, and R. Unbehauen, Stochastic stability of the discrete-time extended Kalman filter, IEEE Transactions on Automatic control, vol. 44, no. 4, pp. 714728, Feb. 1999.

[28] A. J. Rojas and F. Lotero, Signal-to-noise ratio limited output feedback control subject to channel input quantization, IEEE Transactions on Automatic control, vol. 60, no. 2, pp. 475-479, Feb. 2015.

[29] D. Simon and T. Chia, Kalman filtering with state equality constraints, IEEE Transactions on Aerospace and Electronic Systems, vol. 38, no. 1, pp. 128-136, Jan. 2002.

[30] Y. Theodor and U. Shaked, Robust discrete-time minimumvariance filtering, IEEE Transactions on Singnal Processing, vol. 44, no. 2, pp. 181-189, Feb. 1996.

[31] A. Vempaty, L. Tong, and P. Varshney, Distributed inference with Byzantine data: State-of-the-art review on data falsification attacks, IEEE Signal Processing Magazine, vol. 30, no. 5, pp. 65-75, Jan. 2013.

[32] A. Vempaty, O. Ozdemir, K. Agrawal, H. Chen, and P. K. Varshney, Localization in wireless sensor networks: Byzantines and mitigation techniques, IEEE Transactions on Singnal Processing, vol. 61, no. 6, pp. 1495-1508, Mar. 2013.

[33] F. Yang, H. Dong, Z. Wang, W. Ren and F. E. Alsaadi, A new approach to non-fragile state estimation for continuous neural networks with time-delays, Neurocomputing, vol. 197, pp. 205-211, 2016. 
[34] H. Yang, Z. Wang, H. Shu, F. E. Alsaadi and T. Hayat, Almost sure $H_{\infty}$ sliding mode control for nonlinear stochastic systems with Markovian switching and timedelays, Neurocomputing, vol. 175, pp. 392-400, 2016.

[35] Y. Yu, H. Dong, Z. Wang, W. Ren and F. E. Alsaadi, Design of non-fragile state estimators for discrete timedelayed neural networks with parameter uncertainties, Neurocomputing, vol. 182, pp. 18-24, 2016.

[36] N. Zeng, Z. Wang and H. Zhang, Inferring nonlinear lateral flow immunoassay state-space models via an unscented Kalman filter, Science China Information Sciences, vol. 59 no. 11, Art. No. 112204. doi:10.1007/s11432-016-0280-9, 2016.

[37] J. Zhang, R. S. Blum, X. Lu, and D. Conus, Asymptotically optimum distributed estimation in the presence of attacks, IEEE Transactions on Singnal Processing, vol. 63, no. 5, pp. 1086-1101, Mar. 2015.

[38] M. Zhu, and S. Martinez, On the performance analysis of resilient networked control systems under replay attacks, IEEE Transactions on Automatic Control, vol. 59, no. 3, Mar. 2014.

Derui Ding received both the B.Sc. degree in Industry Engineering in 2004 and the M.Sc. degree in Detection Technology and Automation Equipment in 2007 from Anhui Polytechnic University, Wuhu, China, and the Ph.D. degree in Control Theory and Control Engineering in 2014 from Donghua University, Shanghai, China. From July 2007 to December 2014, he was a teaching assistant and then a lecturer in the Department of Mathematics, Anhui Polytechnic University, Wuhu, China.

He is currently an associate professor with the Department of Control Science and Engineering, University of Shanghai for Science and Technology, Shanghai, China. From June 2012 to September 2012, he was a research assistant in the Department of Mechanical Engineering, the University of Hong Kong, Hong Kong. From March 2013 to March 2014, he was a visiting scholar in the Department of Information Systems and Computing, Brunel University London, UK. From June 2015 to August 2015, he was a research assistant in the Department of Mathematics, City University of Hong Kong, Hong Kong. His research interests include nonlinear stochastic control and filtering, as well as multi-agent systems and sensor networks. He has published around 20 papers in refereed international journals. He is a very active reviewer for many international journals.

Zidong Wang was born in Jiangsu, China, in 1966. He received the B.Sc. degree in mathematics in 1986 from Suzhou University, Suzhou, China, and the M.Sc. degree in applied mathematics in 1990 and the Ph.D. degree in electrical engineering in 1994, both from Nanjing University of Science and Technology, Nanjing, China.

He is currently Professor of Dynamical Systems and Computing in the Department of Information Systems and Computing, Brunel University London, U.K. From 1990 to 2002, he held teaching and research appointments in universities in China, Germany and the UK. Prof. Wang's research interests include dynamical systems, signal processing, bioinformatics, control theory and applications. He has published more than 300 papers in refereed international journals. He is a holder of the Alexander von Humboldt Research Fellowship of Germany, the JSPS Research Fellowship of Japan, William Mong Visiting Research Fellowship of Hong Kong.

Prof. Wang serves (or has served) as the Editor-in-Chief for Neurocomputing and an Associate Editor for 12 international journals, including IEEE Transactions on Automatic Control, IEEE Transactions on Control Systems Technology, IEEE Transactions on Neural Networks, IEEE Transactions on Signal Processing, and IEEE Transactions on Systems,
Man, and Cybernetics - Part C. He is a Fellow of the IEEE a Fellow of the Royal Statistical Society and a member of program committee for many international conferences.

Daniel W. C. Ho received the B.S., M.S., and Ph.D. degrees in mathematics from the University of Salford, Manchester, U.K., in 1980, 1982, and 1986, respectively.

He was a Research Fellow with the Industrial Control Unit, University of Strathclyde, Glasgow, U.K., from 1985 to 1988. In 1989, he joined the Department of Mathematics, City University of Hong Kong, Hong Kong. In 2015, he has been appointed as a Chair Professor and an Assistant Dean (Teaching Innovations) of the College of Science and Engineering. His current research interests include estimation and filtering theory, complex dynamical distributed networks, multiagent networks, nonlinear singular systems, and stochastic systems.

Prof. Ho was a recipient of the best paper award from the Eighth Asian Control Conference. In 2012, he received the Chang Jiang Chair Professor Award from the Ministry of Education, China. He is the Subject Editor of the Journal of the Franklin Institute and an Associate Editor of four international control journals, including IET Control Theory and its Applications, and the Asian Journal of Control. He has been named in the list of Highly Cited Researchers 2014 and 2015 published by Thomson Reuters.

Guoliang Wei received the B.Sc. degree in mathematics from Henan Normal University, Xinxiang, China, in 1997 and the M.Sc. degree in applied mathematics and the Ph.D. degree in control engineering, both from Donghua University, Shanghai, China, in 2005 and 2008, respectively.

He is currently a Professor with the Department of Control Science and Engineering, University of Shanghai for Science and Technology, Shanghai, China. From March 2010 to May 2011, he was an Alexander von Humboldt Research Fellow in the Institute for Automatic Control and Complex Systems, University of Duisburg-Essen, Germany. From March 2009 to February 2010, he was a post doctoral research fellow in the Department of Information Systems and Computing, Brunel University, Uxbridge, UK, sponsored by the Leverhulme Trust of the UK From June to August 2007, he was a Research Assistant at the University of HongKong. From March to May 2008, he was a Research Assistant at the City University of Hong Kong.

His research interests include nonlinear systems, stochastic systems, and bioinformatics. He has published more than 20 papers in refereed international journals. He is a very active reviewer for many international journals. 